\title{
Estudo da multiplicação e divisão de números fracionários no currículo de matemática do estado de São Paulo
}

\author{
Angélica da Fontoura Garcia Silva \\ Professora, Uniban/SP \\ angelicafontoura@gmail.com
}

\section{Aparecida Rodrigues Silva Duarte \\ Professora, Uniban/SP - Univás/MG \\ aparecida.duarte6@gmail.com}

\section{Dario Vieira de Oliveira Filho}

Participante, Observatório da Educação Uniban/SP

dario.volfi@bol.com.br

\section{Resumo}

Este estudo tem por finalidade analisar os pressupostos e diretrizes que alicerçam o atual currículo de Matemática proposto para o estado de São Paulo, no que se refere à introdução da multiplicação e da divisão com frações e como tais orientações têm sido interpretadas pelos professores. Fundamenta-se em pesquisas que versam sobre a formação de professores, bem como em estudos que investigam questões didáticas sobre o objeto matemático em questão. Verificou-se que o currículo incorporou alguns dos resultados de pesquisas sobre esse tema, mas não apresentou uma discussão sobre os diferentes significados da multiplicação e da divisão de frações.

Palavras-chave: Educação matemática. Formação de professores. Currículo. Multiplicação e divisão com frações. Conhecimento profissional docente.

\section{Study of multiplication and division of fractional numbers in mathematics' curriculum of São Paulo state}

\begin{abstract}
This study pretends to analyze the premises and guidelines that underpin the current Mathematics curriculum proposed for São Paulo state regarding the introduction of multiplication and division with fractions and how such guidelines have been interpreted by teachers. This quest is based on researches which focused on teachers' training as well as in studies that investigated didactic questions about the mathematical object in discussion. It was ascertained that the curriculum has incorporated some of the results pointed on researches in this topic, but it did not present a discussion about the different meanings of multiplication and division of fractions.
\end{abstract}

Keywords: Mathematics education. Teacher training. Curriculum. Multiplication and division with fractions. Teacher's professional knowledge. 


\section{Introdução}

Neste artigo, analisamos os pressupostos e diretrizes que alicerçam o atual currículo de Matemática proposto para o estado de São Paulo, Brasil, no que se refere à introdução da multiplicação e da divisão de números racionais em sua representação fracionária ${ }^{1}$, com o propósito de identificar a relação entre as indicações recomendadas pelo movimento de implementação curricular e outras orientações de pesquisas que tratam da mesma temática. Procuramos ainda, analisar as interpretações que professores de matemática da rede pública estadual de São Paulo, que lecionam no Ensino Fundamental e Médio, fazem sobre o ensino dessas operações e como interpretam as orientações propostas no material de apoio ao currículo - o Caderno do Professor. Para delimitar nosso problema de pesquisa, notadamente de cunho qualitativo-bibliográfico, apresentamos a relevância do tema, a fundamentação teórica que utilizamos em nossa análise, os pressupostos do Currículo Oficial do Estado de São Paulo e os estudos que vêm sendo desenvolvidos. Em seguida, apresentamos a análise e considerações finais.

\section{Relevância e Fundamentação Teórica}

Julgamos que essa temática seja relevante, pois a compreensão do conceito de fração constitui etapa importante para a ampliação da ideia de número, já que essa exige do estudante a reelaboração de noções relativas aos números naturais, além de permitir o desenvolvimento de ideias importantes ligadas às operações algébricas elementares. Trata-se de um conceito, cuja compreensão parece não ser evidente, uma vez que avaliações institucionais e diversas pesquisas nacionais e internacionais apontam-no como de difícil entendimento, tanto para alunos como para professores.

No que se refere aos processos de ensino e aprendizagem das frações, observamos a existência de um grande número de pesquisas. Mack (1990), por exemplo, no início da década de 90, realizou uma pesquisa, nos Estados Unidos, com estudantes na faixa etária de 11 a 12 anos, com o objetivo de verificar se eles conseguiam construir o conceito de fração a partir dos seus conhecimentos informais. $\mathrm{O}$ autor mostra que muitos alunos sem conhecimento prévio da fração conseguiram resolver problemas ligados ao cotidiano utilizando-se, predominantemente, de conhecimentos informais. Porém, os alunos que tinham alguma experiência em operar com frações, concentravam-se essencialmente nas manipulações simbólicas e encontraram, segundo a autora, mais dificuldade em "dar sentido aos símbolos e procedimentos da fração" (MACK, 1990,

1. Neste artigo utilizaremos o termo fração para designar a representação fracionária dos Números Racionais. 
p. 34). Em momento algum o autor admite que o problema seja insuperável, no entanto, chama a atenção para a necessidade de "uma grande dose de tempo e sensibilidade por parte do professor" para superá-lo (MACK, 1990, p. 34). Assim sendo, Mack defende um argumento em favor de propostas que priorizem vivencias que possibilitem a construção dos conceitos antes de apresentar os procedimentos aos estudantes.

A esse respeito, Pinto e Monteiro (2008) acrescentam que: “A compreensão da multiplicação e divisão com números inteiros, assim como as noções básicas de frações são fundamentais para se avançar para a multiplicação e divisão de números fracionários" (p. 201). Entretanto, alguns autores constatam que, ainda hoje, bem como no passado, o ensino das operações está muito centrado no procedimento.

Nesse sentido, Neves (2008) analisou 42 pesquisas brasileiras e internacionais, publicadas no período de 1999 a 2006, quando observou que, em geral, os professores pesquisados valorizavam os "aspectos procedimentais e suas concepções mostram-se altamente influenciadas pelas experiências vividas como alunos na educação básica" (NEVES, 2008, p. 93).

Quanto ao ensino, Neves (2008) igualmente observou que, nas 42 pesquisas publicadas, "(...) de modo consensual, os erros e as dificuldades com a divisão estiveram presentes nas resoluções da maioria dos alunos, tantos os das séries iniciais quanto das séries finais do Ensino Fundamental" (NEVES, 2008, p. 91).

Assim, tudo indica haver uma relação entre as dificuldades encontradas pelos estudantes e a preferência dos docentes pela abordagem procedimental ao ensinar a multiplicação e divisão com números racionais. Além disso, outras pesquisas brasileiras também apontam a existência de uma relação intrínseca das concepções dos professores com a própria aprendizagem e a sua influência sobre o ensino e aprendizagem dos alunos (CAMPOS et al., 1995; CANOVA, 2006; DAMICO, 2007; GARCIA SILVA, 2007).

Dentre esses estudos, destaca-se aquele realizado por Damico (2007). O autor investigou a formação inicial de professores de matemática para o ensino das frações em duas universidades localizadas no estado de São Paulo. O pesquisador observou um acentuado desequilíbrio entre o conhecimento conceitual e processual, sendo que o último prevaleceu. Verificou, também, um conhecimento didático relacionado às formas de representação dos números racionais aquém do que normalmente é ensinado no Ensino Fundamental.

A investigação realizada por Oliveira Filho (2011) teve por objetivo analisar as concepções, sobre o ensino dos números racionais na representação fracionária, de professores de matemática que lecionam no sexto e sétimo anos do Ensino Fundamental e integrantes de um Projeto denominado "Observatório da Educação", financiado pela Coordenação de Aperfeiçoamento de Pessoal de Nível Superior 
(CAPES) e pelo Instituto Nacional de Estudos e Pesquisas Educacionais Anísio Teixeira (INEP). Verificou-se que os professores participantes da pesquisa ${ }^{2}$ introduziam o tema, fundamentalmente, por meio do significado parte-todo. Ademais, esses docentes faziam uso de representações gráficas para introduzir a noção de equivalência de frações e justificar as operações de adição e subtração de frações. Entretanto, não procuravam justificar os procedimentos que envolviam as operações de multiplicação e divisão de frações e os introduziam por meio de algoritmos.

Essas constatações foram igualmente verificadas em estudos internacionais. Armstrong e Bezuk, em 1995, afirmavam que o foco da aprendizagem dos professores por eles investigados também era procedimental, fato que não favorecia a compreensão conceitual de seus alunos. Os autores observavam o que Borko, Eisenhart, Brown, Underhill, Jones, e Agard já haviam constatado, em 1992, quando analisaram um episódio de ensino de multiplicação e divisão de frações: que professores, assim como a maioria dos adultos, tinham “( ...) uma compreensão limitada do significado da multiplicação e divisão de frações” (apud ARMSTRONG; BEZUK, 1995, p. 87).

Do mesmo modo, ao analisar o conhecimento de professores americanos e chineses, Ma (2009), verificou, por exemplo, que "embora $43 \%$ dos professores americanos tivessem conseguido calcular corretamente uma resposta completa [para uma divisão de frações], nenhum mostrou entendimento da fundamentação lógica subjacente aos cálculos" (2009, p.154).

Enfim, analisando as pesquisas apresentadas, há indícios de que as operações com fração, e em especial, a multiplicação e divisão de frações, não estão sendo desenvolvidas e compreendidas como deveriam. Nessa perspectiva, a insuficiência de um conhecimento profissional docente sobre a multiplicação e divisão de frações é preocupante.

Estudos como os de Shulman $(1986$; 1987) consideram a importância do conhecimento do conteúdo, enfatizando a necessidade do professor dominá-lo, sob pena de comprometer o ensino. É no processo de desenvolvimento profissional docente, em especial, na formação inicial e continuada, que se pode favorecer a ampliação desse conhecimento. Ainda para Shulman (1986; 1987), os docentes devem possuir três tipos de conhecimento: Conhecimento da matéria ensinada; Conhecimento pedagógico do conteúdo e Conhecimento curricular.

2. Os sujeitos desse estudo eram participantes da formação continuada desenvolvida pelo Programa de Pós-Graduação em Educação Matemática da Universidade Bandeirante de São Paulo, no âmbito do projeto deminado Observatório da Educação. A referida pesquisa foi aprovada pelo Comitê de Ética da instituição de ensino responsável pela investigação e contou com a autorização dos professores participantes para utilização dos dados enquanto situação de análise. Durante as entrevistas, como integrantes do corpo docente de escolas públicas paulistas, os professores participantes discorreram sobre suas práticas em sala de aula. Essas entrevistas estão disponíveis, na íntegra, no estudo de Oliveira (2011). 
O Conhecimento pedagógico do conteúdo é o que mais tem chamado atenção dos pesquisadores que discutem a prática do professor, por tratar-se de um conhecimento que combina o conteúdo com o modo de ensiná-lo, pois, conforme esclarece Shulman, o conhecimento pedagógico do conteúdo

[...] incorpora os aspectos do conteúdo mais relevantes para serem ensinados. Dentro da categoria de conhecimento de conteúdo pedagógico, incluo, para a maioria dos tópicos regularmente ensinados de uma área específica de conhecimento, as representações mais úteis de tais idéias, as analogias mais poderosas, ilustrações, exemplos, (...) as concepções e preconcepções que estudantes de diferentes idades e repertórios trazem para as situações de aprendizagem (SHULMAN, 1986, p. 9).

Trata-se, portanto, de um conhecimento específico da atividade de um professor que desempenha importante papel nos processos de ensino e aprendizagem.

No que tange à relação entre o Conhecimento Profissional Docente e os processos de ensino e aprendizagem, entende-se que a análise de documentos que orientam professores, juntamente com a análise de suas percepções sobre esses documentos, podem auxiliar na compreensão do processo de formação docente, pois, muitas vezes, o professor utiliza-se de tais indicações para preparar suas aulas.

O cenário de inovação curricular, vivenciado pelas escolas públicas paulistas desde 2008, apresenta elementos que podem favorecer as referidas análises, uma vez que, no bojo desse novo currículo de matemática, há indicações de materiais orientadores, os quais são apresentados desde os fundamentos que o permeiam, até orientações mais específicas para o desenvolvimento de conteúdos.

Como destaca Pietropaolo (2002), há uma relação intrínseca entre o processo de inovação curricular e a formação docente. Para o autor, tais orientações poderiam:

[...] nortear a formação inicial e continuada de professores, pois à medida que os fundamentos do currículo se tornem claros, fica implícito o tipo de formação que se pretende para o professor, como também orientar a produção de livros e de outros materiais didáticos, contribuindo dessa forma para configuração de uma política voltada à melhoria do ensino fundamental (PIETROPAOLO, 2002, p. 37).

Dessa maneira, torna-se fundamental que, em nossa análise, identifiquemos a existência ou não de uma relação entre as constatações veiculadas nas pesquisas e as indicações divulgadas no "Caderno do Professor", um dos principais documentos 
de apoio ao currículo de matemática, que contém indicações pedagógicas a serem desenvolvidas pelo professor nas escolas públicas do estado de São Paulo, no tocante ao ensino do conceito da multiplicação e divisão com frações.

\section{O Currículo Oficial do Estado de São Paulo}

O novo currículo, implementado desde 2008, foi intitulado inicialmente de Proposta Curricular do Estado de São Paulo, e a partir do ano de 2009, como Currículo do Estado de São Paulo.

Para garantir o processo de mudança curricular, foram elaborados materiais de apoio: Cadernos do Professor (CP) e Cadernos do Aluno (CA); ambos organizados por bimestre. O CP apresenta orientações metodológicas e sugestões de aulas, materiais complementares, de avaliação e recuperação; já o CA é considerado um complemento ao $\mathrm{CP}$, contendo algumas das propostas indicadas no material do professor.

A introdução presente no $\mathrm{CP}$ menciona que o currículo foi organizado tendo como foco a educação centrada em competências ${ }^{3}$ e na contextualização, levando-se em conta "a democratização da escola", no sentido de garantir sua unidade e sua a diversidade.

Quanto ao elenco de conteúdos apresentado no material, observamos, num primeiro momento, que ele não se diferencia de forma substancial do que culturalmente é ensinado para este nível de ensino. Tais conteúdos foram distribuídos por bimestres para cada série/ano, sendo organizados em três blocos temáticos: Números, Geometria, e Relações, onde cada um está presente direta ou indiretamente na lista dos conteúdos. Todavia, as orientações contidas nesse documento chamam a atenção para o fato de que ao se desenvolver os temas deve-se explorar "ideias fundamentais: proporcionalidade, equivalência, ordem e aproximação".

\section{A multiplicação e divisão de frações}

As orientações contidas no "Caderno do Professor" sugerem que a introdução do conteúdo frações ocorra no $1^{\circ}$ bimestre do $7^{\circ}$ ano do Ensino Fundamental (crianças

3. Utilizam-se, nesse documento introdutório do Currículo, como competências básicas, aquelas indicadas pelo Exame Nacional do Ensino Médio (Enem). Elas estão associadas aos três eixos: capacidade de expressão em diferentes linguagens, incluídas a língua materna, a Matemática, as artes, entre outras; à capacidade de compreensão de fenômenos, que incluem desde a leitura de um texto até a "leitura" do mundo; à capacidade de contextualizar, de enfrentar situações-problema; à capacidade de argumentar de modo consistente, de desenvolver o pensamento crítico; e à capacidade de decidir (SÃO PAULO, 2008, p.19). 
de 11 e 12 anos de idade) por meio de situações envolvendo a ideia de medida, pois se considera que os estudantes já haviam trabalhado números naturais e suas operações bem como a representação fracionária. (SÃO PAULO, 2009).

Os autores também recomendam aos professores que realizem um diagnóstico a fim de avaliar o conhecimento dos alunos sobre frações, no que diz respeito à nomenclatura e à representação parte-todo de uma figura. Os professores devem, igualmente, atentar para a necessidade de desenvolver habilidades que possibilitem aos estudantes "reconhecer a diferença entre os números naturais, diretamente ligados à ideia de contagem e ordenação, e os números fracionários relacionados aos processos de medida" (SÃO PAULO, 2009, p. 9). Nesse sentido, o documento em questão infere que provavelmente os professores dos níveis de ensino anteriores já haviam desenvolvido trabalhos com frações utilizando-se do significado parte-todo.

Reforçando essa ideia, pesquisas como as de Nunes e Bryant (1997), tomando como base estudos de Campos et al. (1995), destacam que há uma forte tendência, por parte dos docentes, em trabalhar o conceito de fração utilizando principalmente o significado parte-todo. De modo similar, outras pesquisas verificaram a mesma prática do professor, tais como os Parâmetros Curriculares Nacionais (PCN, 1998), Garcia Silva (2007), Campos e Magina (2006), Canova (2006) dentre outros.

Destaca-se que as referidas pesquisas, bem como as orientações oficiais contidas no CP, defendem a importância de que os estudantes desse nível de ensino ampliem o conhecimento dos significados desses conceitos, de modo a perceber a necessidade da utilização de frações em seus diferentes significados e situações.

As operações multiplicação e divisão de frações são introduzidas na seção "Situação de Aprendizagem 3"; e tem-se por objetivo habilitar o aluno a operar por meio do reconhecimento e interpretação dessas operações utilizando-se de recursos, como a partição de barras. Esse documento enfatiza a importância dos estudantes saberem trabalhar com destreza a multiplicação e a divisão de fração (SÃO PAULO, 2009).

O CP inicia o assunto apresentando a multiplicação de um número inteiro por uma fração, indicando que tal operação é análoga ao produto de dois números naturais.

Traz, como exemplo, a multiplicação de 4 por $\frac{1}{3}$. Para tanto, recomenda que o professor apresente essa multiplicação como: 4 × $\frac{1}{3}=\frac{1}{3} \times 4=\frac{1}{3}+\frac{1}{3}+\frac{1}{3}+\frac{1}{3}$, ou seja, que calcular $\frac{1}{3}$ de 4 é o mesmo que calcular a terça parte de quatro. Além disso, enfatiza que esse mesmo procedimento é válido para a multiplicação entre duas frações. 
No procedimento de multiplicar um número natural por uma fração, o CP faz uso do mesmo recurso utilizado nos anos anteriores, quando trata do produto de dois números naturais, desenvolvendo o conceito de uma soma de parcelas e destacando a propriedade comutativa.

O caderno também relaciona a preposição "de" com a multiplicação, destacando a validade da propriedade comutativa, ou seja, que se pode calcular $\frac{3}{4}$ de $\frac{4}{5}$ ou $\frac{4}{5}$ de $\frac{3}{4}$, obtendo o mesmo resultado.

Em seguida, o CP trata do produto de duas frações. Para tanto, utiliza-se da representação na forma geométrica. Essa mesma metodologia foi utilizada anteriormente, tanto na Proposta Curricular de São Paulo de 1987, como nos Parâmetros Curriculares de 1998 e no material de apoio (CP) do Currículo do Estado de São Paulo de 2009.

\section{Proposta Curricular de São Paulo (1987, p.82)}

Multiplicação de frações

Quanto à multiplicação de frações, estamos, pela primeira vez, diante de uma operação cujo conceito tem que ser modificado, em relação ao que foi feito com números naturais. Não cabe mais, aqui, considerar a multiplicação como uma soma reiterada de parcelas iguais. Nesse caso pode-se dar um significado à operação de multiplicação de frações, pensando-se em "partes de partes" do total considerado, isto é: $\frac{2}{5} \times \frac{3}{4}$

Seria trabalhando como: procurar $\frac{2}{5} \operatorname{dos} \frac{3}{4}$ de um todo

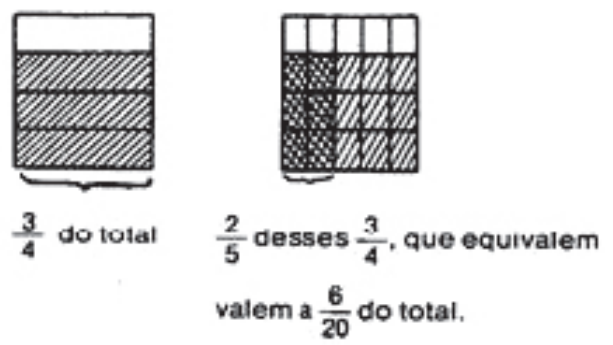

ou seja: $\frac{2}{5} \times \frac{3}{4}=\frac{6}{20}$ 
A partir de várias experiências como esta, os alunos irão, cada um dentro de seu ritmo, chegando a uma regra prática para encontrar o resultado. Para esse trabalho, é necessário associar o símbolo $\mathrm{X}$ à situação de partes de algum total. Pode-se iniciar isso, trabalhando as ideias de dobro, triplo, etc.

\section{Parâmetros Curriculares Nacionais - PCN (1998, p. 104)}

A compreensão da multiplicação com frações pode ser pensada como "partes de partes do total" (neste caso a multiplicação não se apóia de adição reiterada). Assim, $\frac{2}{5} x \frac{3}{4}$ pode ser interpretado como procurar $\frac{2}{5}$ dos $\frac{3}{4}$ de um todo.
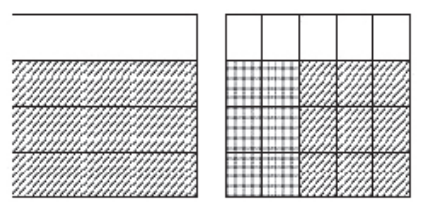

$$
\frac{2}{5} \times \frac{3}{4}=\frac{6}{20}
$$

A partir de várias experiências como essas, os alunos poderão construir um procedimento para multiplicar frações.

Observe-se também que é possível explicar a multiplicação de decimais pela multiplicação de frações. Exemplo:

$$
0,12 \times 0,8=\frac{12}{100} \times \frac{8}{10}=\frac{12 \times 8}{100 \times 10}=\frac{96}{1000}=0,096
$$

\section{Material de Apoio - CP}

\section{Currículo São Paulo (2009, p.32)}

Utilizamos retângulos para representar a unidade e, em seguida, os dividiremos em 4 partes iguais (marcando 3 ) e em 5 partes iguais (marcando 4). Se quremos $\frac{3}{4} x \frac{4}{5}$, então estamos interessados em encontrar $\frac{3}{4} d e \frac{4 "}{5}$, ou seja, devemos pegar $\frac{4}{5}$ da representação correspondente aos $\frac{3}{4}$, o que pode ser obtido por uma intersecção, como mostra a sequência de figuras: 


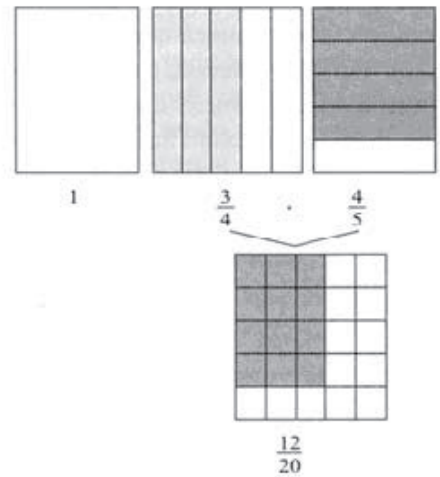

$\mathrm{Na}$ contagem final de quadradinhos para representar a fração resultante da operação, $\frac{12}{20}$, o numerador 12 foi obtido do produto das colunas marcadas em $\frac{3}{4}$ pelas linhas marcadas em $\frac{4}{5}$, ou seja, pelo numerador da primeira fração e o numerador da segunda.

Analisando as três indicações podemos observar que essas propostas apresentam orientações semelhantes para o trabalho do professor quando anunciam a multiplicação como "parte de parte", conforme já indicava o currículo de São Paulo da década de 1980, o PCN de 1998 e o CP de 2009.

Notamos que, apesar das insistências das prescrições oficiais em recomendar diferentes representações para trabalhar com as operações de multiplicação com frações, estudos recentes mostram que os professores continuam a enfatizar o algoritmo como base para o ensino desse conteúdo matemático.

Vejamos os depoimentos presentes em Oliveira Filho (2011):

Para multiplicar uma fração por outra, multiplico em linha, numerador por numerador e denominador por denominador [...] não uso o material. (PROFESSOR A apud OLIVEIRA FILHO, 2011, p.164).

Na multiplicação, falo para multiplicar o numerador pelo numerador e o denominador pelo denominador (PROFESSOR B apud OLIVEIRA FILHO, 2011, p 167). 
Não faço representação com figuras e nem uso outro recurso para tratar multiplicação e divisão. (PROFESSOR B apud OLIVEIRA FILHO, 2011 p.167).

Na multiplicação, ensino os alunos a multiplicarem em linha, numerador por numerador e denominador por denominador, [...] em nenhum dos dois casos uso qualquer forma de figura ou material concreto, só uso giz e lousa. (PROFESSOR E apud OLIVEIRA FILHO, 2011 p.175).

Analisando as declarações de professores de matemática que lecionam no sexto e sétimo anos do Ensino Fundamental e que utilizavam o CP sobre o ensino das frações entrevistados por Oliveira Filho (2011), observamos que esses educadores indicaram fazer a introdução da multiplicação e divisão de frações apenas por intermédio dos algoritmos. Entretanto, pesquisas como as de Armstrong e Bezuk (1995), por exemplo, indicam que o trabalho focado nos procedimentos não proporciona aos alunos experiências de aprendizagem que lhes permitam adquirir a compreensão necessária do conteúdo de frações.

No tocante à divisão de fração, o caderno inicia o estudo apresentando uma situação-problema que envolve a divisão de $\frac{3}{4}$ por $\frac{2}{3}$, que pode ser representado por $\frac{\frac{3}{4}}{\frac{2}{3}}$ ou $\frac{3}{4}: \frac{2}{3}$.

Oproblemaé apresentado da seguinte maneira: $\mathrm{Se} \frac{2}{3}$ de uma lata de tinta dão para pintar $\frac{3}{4}$ de uma parede, que fração da parede conseguirei pintar com uma lata de tinta inteira?

Esse questionamento vem acompanhado da seguinte representação:

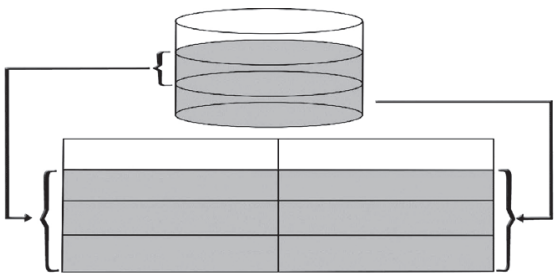

(SÃO PAULO, 2009, p. 33) 
No material de apoio há a discussão da resolução. Inicia-se comentando que dividindo a lata de tinta em 3 partes iguais, o problema nos diz que 2 delas foram utilizadas. Dividindo-se a parede em 4 partes iguais (linhas horizontais na figura), e subdividirmos cada parte da parede em 2 (pois foram utilizadas 2 partes, se são usadas duas partes de tinta), a parede é dividida em $4.2=8$ partes. Portanto, cada parte de tinta permite pintar 3 dessas partes da parede. Logo, a lata inteira, que tem 3 partes, permite pintar $3 \times 3=9$ das partes da parede. Assim, a fração da parede pintada será

igual a $\frac{\frac{3}{4}}{\frac{2}{3}}=\frac{3}{4} \times \frac{3}{2}=\frac{9}{8}$, em que 4.2 é o número de partes em que foi dividida a parede, e $3 \times 3$ é o total das partes que serão pintadas, usando-se a lata inteira.

Com $\frac{3}{4} \times \frac{3}{2}$, obtivemos uma expressão com produto de frações, que é equivalente a expressão inicial de divisão de frações.

Em seguida, e finalizando o assunto, o CP apresenta uma lista contendo 4 problemas envolvendo divisão de frações, sem, no entanto, apresentar uma reflexão sobre a importância de trabalhar outros significados da operação divisão.

Essa atividade, segundo os autores, apresenta uma forma de desenvolver a divisão de frações para justificar a maneira mecânica utilizada nessa operação, qual seja, "multiplicar a primeira fração pelo inverso da segunda fração", utilizando a ideia de divisão por partilha, uma vez que o problema investiga o valor correspondente ao valor unitário do divisor. Vale ressaltar que autores como Pinto e Monteiro (2008) entendem que "estas situações de partilha permitem fazer uma ligação à divisão dos números inteiros e não devem ser postas de lado quando se desenvolve o conceito de divisão de números racionais" (2008, p. 212).

Assim como as autoras, consideramos que essa situação, que é a mais trabalhada com os números Naturais, não é tão imediata para as frações. No caso do divisor ser um número fracionário, a dificuldade reside no fato de que não se pretende "partilhar" por um número inteiro.

Nesse mesmo sentido, Pinto e Monteiro (2008) defendem a importância de oferecer aos alunos experimentações em que se apresentem problemas envolvendo o significado da partilha de frações e que esse estudo deve ser ampliado utilizando situações que envolvam a divisão como medida e a divisão como operação inversa da multiplicação. Segundo as autoras, "é fundamental que os professores possuam um 
conhecimento bastante aprofundado da divisão, na sua relação com a multiplicação e das suas propriedades" (2008, p. 218).

Todavia não observamos no material de apoio $(\mathrm{CP})$ uma preocupação em oferecer argumentos de forma a convencer o professor sobre a importância da utilização de situações-problema envolvendo os diferentes significados da divisão com frações. Nos depoimentos a seguir, podemos observar que a apresentação dessa situação parece não ter encontrado eco dentre os docentes:

Na divisão, conservo a primeira fração e multiplico pelo inverso da segunda, não uso o material. (PROFESSOR A apud OLIVEIRA FILHO, 2011, p.164).

Na divisão de uma fração por outra... conservo a primeira fração (a de cima) e multiplico pelo inverso da fração de baixo (que está no denominador). (PROFESSOR B apud OLIVEIRA FILHO, 2011, p. 167).

Não faço representação com figuras e nem uso outro recurso para tratar multiplicação e divisão. (PROFESSOR B apud OLIVEIRA FILHO, 2011, p.167).

Na divisão, conservo a primeira fração e inverto a segunda, trocando de lugar o numerador com denominador e multiplico em linha; em nenhum dos dois casos uso qualquer forma de figura ou material concreto só uso giz e lousa. (PROFESSOR E, apud OLIVEIRA FILHO, 2011 p.175).

Tais respostas revelam, a nosso ver, que, por ocasião da aplicação da entrevista, no ensino de divisão com frações, os professores deram ênfase ao algoritmo "inverter o divisor e multiplicar", não explorando o trabalho com diferentes significados dessa operação.

Analisando as orientações contidas no $\mathrm{CP}$, observamos que não havia, por parte dos autores do documento, uma reflexão sobre como trabalhar com todos os significados da divisão de forma a auxiliar os professores no entendimento desses significados em diferentes contextos.

\section{Considerações Finais}

Embora as prescrições oficiais, notadamente a Proposta Curricular de São Paulo (1987) os PCN (1998) e o Caderno do Professor (2009), recomendem enfaticamente trabalhar as operações de multiplicação e divisão com diferentes representações e que algumas investigações notem que o trabalho voltado para os procedimentos 
não promovem o desenvolvimento da compreensão do conteúdo das frações, os professores participantes da pesquisa não apresentaram explicações para o trabalho com a multiplicação e divisão de frações. Verificou-se que preferiram introduzi-las por meio de algoritmos.

Alguns pesquisadores, como Pinto e Monteiro (2008), defendem a importância de oferecer aos alunos experimentações que apresentem problemas que recorram ao significado da partilha de frações e que os professores tenham um conhecimento aprofundado sobre a operação divisão e da sua relação com a multiplicação e suas propriedades. Entretanto, observou-se que, o material de apoio analisado não se empenhou em enfatizar sobre a importância do uso do conhecimento adquirido em situações-problema que demandem a utilização dos diferentes significados da multiplicação e divisão com frações. O material de apoio não ofereceu, por exemplo, elementos que permitisse ao leitor aprofundar seus conhecimentos sobre a conexão entre o significado da divisão e o uso compreensivo do algoritmo da divisão "inverter o divisor e multiplicar".

Nessa perspectiva, analisando as pesquisas citadas e considerando, assim como Ponte (1992), que as concepções dos alunos são diretamente influenciadas pela prática docente, acreditamos ser importante atentar para o conhecimento docente, pois este tem papel fundamental no processo de ensino e aprendizagem.

Sob o ponto de vista de Shulman (1986), a falta de domínio do conteúdo operações de multiplicação e divisão de frações implicaria também na ausência de Conhecimento Pedagógico do Conteúdo e Curricular. Dessa forma, a falta de compreensão sobre os significados da multiplicação e divisão de frações parece limitar a seleção e organização de tarefas utilizadas pelo professor.

\section{Referências}

ARMSTRONG, B.; BEZUK, N. Multiplication and division of fractions: The search for meaning. In: SOWDER J. T.; SCHAPPELLE, B. P. (Eds.). Providing a foundation for teaching mathematics in the middle grades. p. 85-119, Albany: New York Press, 1995.

\section{BRASIL. Parâmetros Curriculares Nacionais para o Ensino Fundamental: Matemática. Brasília: MEC/SEF, 1998.}

CAMPOS, T. et al. Lógica das equivalências. Relatório de pesquisa não publicado. São Paulo: PUC. 1995. 
CAMPOS T.; MAGINA, S. Primary school teachers' concepts of fractions and teaching strategies. Anais... ICME 10. Copenhague, 2006. Disponível em: <www. icmeorganisers. dk/tsg22/ campos\% 20and\%20magina.doc.>. Acesso em: jan. 2009.

CANOVA, R. F. Crença, concepção e competência dos professores do $1^{\circ}$ e $2^{\circ}$ ciclos do ensino fundamental com relação à fração. Dissertação (Mestrado). PUC/SP. São Paulo, 2006.

DAMICO, A. Uma investigação sobre a formação inicial de Professores de Matemática para o ensino de números racionais no ensino fundamental. Tese (Doutorado em Educação Matemática). PUC/SP. São Paulo, 2007.

GARCIA SILVA, A. F. O desafio do desenvolvimento profissional docente: análise da formação continuada de um grupo de professores das séries iniciais do ensino fundamental, tendo como objeto de discussão o processo de ensino e aprendizagem das frações. Tese (Doutorado em Educação Matemática). PUC/SP. São Paulo, 2007

MA, L. Aprender e Ensinar Matemática Elementar. Lisboa: SPM/Gradiva, 2009.

MACK, N.K. Learning fractions with understanding building on informal knowledge. Journal for Research in Mathematics Education, v.21, p.16-32, 1990.

NEVES, R. S. P. A divisão e os números racionais: uma pesquisa de intervenção psicopedagógica sobre o desenvolvimento de competências conceituais de alunos e professores. Tese (Doutorado). Universidade de Brasília, Brasília, 2008.

NUNES, T.; BRYANT, P. Crianças Fazendo Matemática. Porto Alegre: Artes Médicas. 1997.

OLIVEIRA FILHO, D. Concepções de professores da rede pública estadual de São Paulo acerca do ensino das frações no ensino fundamental. Dissertação (Mestrado em Educação Matemática). Uniban, São Paulo, 2011.

PIETROPAOLO, R. Parâmetros Curriculares de Matemática para o Ensino Fundamental. Educação Matemática em Revista. São Paulo: SBEM, ano 9, n. 11A, p. 34-8, abr. 2002. 
PINTO, H.; MONTEIRO, C. A. Divisão de números racionais. In. Brocardo, J. Serrazina, L. Rocha, I. O sentido do número: reflexões que entrecruzam teoria e prática. Lisboa: Escolar, 2008.

PONTE, Concepções dos professores de Matemática e processos de formação. In: Educação Matemática: Temas de investigação Educacional. Lisboa: Instituto de Inovação, 1992, p. 185-239.

SÃO PAUlO (Estado). Proposta Curricular do Estado de São Paulo: Matemática. São Paulo: SEE, 2008.

. Caderno do professor: matemática, ensino médio $-6^{\mathrm{a}}$ série $/ 7^{\circ}$ ano $1^{\mathrm{o}}$ bimestre. São Paulo: SEE, 2009.

SHULMAN, L. Those who understand: knowledge growth in teaching. Educational Researcher, 15 (2), p. 4-14, 1986.

. Knowledge and teaching foundations of the new reform. Educational Rewiew, 57 (1), p. 1-22, 1987.

Submetido em maio de 2012 Aprovado em setembro de 2012 